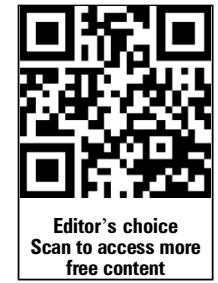

Department of Obstetrics and Gynecology, Melaka Manipal Medical College, Melaka, Malaysia

\section{Correspondence to}

Dr Kavitha Nagandla, Department of Obstetrics and Gynecology, Melaka Manipal Medical College, Jalan Batu Hampar, Bukit Baru, Melaka 75150, Malaysia; kavitha.nagandla@gmail.com

Received 19 November 2012 Revised 24 January 2013 Accepted 3 March 2013 Published Online First 22 March 2013
To cite: Nagandla K, De S. Postgrad Med J

2013;89:402-410.

\title{
Restless legs syndrome: pathophysiology and modern management
}

\author{
Kavitha Nagandla, Somsubhra De
}

\section{ABSTRACT}

Restless legs syndrome (RLS) is a common sensory motor neurological disorder that is characterised by an irresistible urge to move the legs that significantly affects the quality of life of the patient. Prevalence in the general population is $5-25 \%$ and it is twice as prevalent in women as in men. RLS is the most common movement disorder in pregnancy with a fourfold increased risk of developing this disorder later in life. The pathophysiology of RLS is centred on dopaminergic dysfunction, reduced central nervous system iron, genetic linkages, or alteration in neurotransmitters such as hypocretins, endorphins levels and immune dysfunction and inflammatory mechanisms. With the emergence of new evidence, there are changes to the previous treatment recommendations for RLS. There is sufficient evidence to conclude that dopamine agonists such as rotigotine transdermal patch,

pramipexole, ropinirole, gabapentin enacarbil, pregabalin and gabapentin are effective in the short-term treatment of RLS and rotigotine, followed by gabapentin enacarbil, ropinirole, pramipexole and gabapentin for long-term treatment. Based on expert consensus, the recommendation for daily RLS is dopamine agonists or gabapentin or low-potency opioids. Levodopa is less preferred for treating daily RLS due to its high risk of augmentation. For intermittent RLS, it is levodopa or dopamine agonists or low-potency opioids or benzodiazepines. For refractory RLS, the choice is to change to gabapentin or a different dopamine agonist, addition of a second agent like gabapentin or benzodiazepine to the existing drug or changing to a high-potency opioid or tramadol. Medications with safety record in pregnancy include opioids and antiepileptics such as carbamazepine and gabapentin. There are concerns that patients with RLS are at risk for metabolic deregulation, autonomic dysfunction and cardiovascular morbidity. However, a recent study concluded that RLS is not associated with increased risk of cardiovascular complications.

\section{INTRODUCTION}

Restless legs syndrome (RLS), also known as Willis-Ekborn disease, ${ }^{1}$ is a sensory motor disorder defined as an urge to move the legs that may be accompanied by dysesthesias, which is referred to as an unpleasant, abnormal sensation that appears without any apparent sensory stimulation. These sensations are predominant when at rest in the evening and night, and are relieved by movement. It can be summarised as 'movement-responsive quiescegenic nocturnal focal akathisia usually with dysesthesias. ${ }^{2}$ Although the diagnosis is predominantly based on the clinical features, poor recognition of symptoms or bizarre description of symptoms often leads to delay in the diagnosis or misdiagnosis of this condition. If RLS is undiagnosed or undertreated, it can lead to severe impairment of various functions that significantly affects the quality of life of these patients. The consequences of severe RLS are related to the sleep disruption that has a negative impact on the daily activities of patients. It is estimated that most RLS patients sleep for an average of $5 \mathrm{~h}$ a day. The consequences of sleep loss are daytime drowsiness, lack of concentration, depression, difficulties with work and disruption of personal and professional relationships. ${ }^{3}$ There is considerable progress made in understanding this disorder and numerous controlled studies are available with new and existing drugs, thereby providing revised recommendations in the prescription practices. The purpose of this review is to provide an update on the current understanding of the pathophysiology of the disease and to highlight the clinical and scientific advances in the field of diagnosis and therapeutic strategies of this disease.

\section{EPIDEMIOLOGY \\ Prevalence}

The prevalence of RLS varies among different population surveyed and the severity of symptoms essential for the diagnosis. Mild symptoms of RLS are identified in about $5-15 \%$ of general population. ${ }^{4}$ The data from REST (RLS Epidemiology, Symptoms and Treatment), which is the largest trial till date with 23052 patients presenting to a primary care centre in the USA revealed that any degree of RLS symptoms is present in $11.9 \%$, with about $9.5 \%$ of them experiencing symptoms that disrupt their daily activities and impair the quality of life. The study group reported that $88 \%$ experienced sensory symptoms and $76 \%$ reported motor disturbances. ${ }^{6}$ RLS occurs in children with an incidence of $2 \%$. The incidence is high among children whose biological parents are affected with RLS. ${ }^{7}$ In another study by Allen $e t$ al, it was observed that the prevalence in women is twice that in men and that it increases up to the age of 79 years and then declines progressively. ${ }^{8}$ The study of the prevalence in East Asia includes a Singaporean survey with estimates of $0.1 \%$ and a Korean survey of $12.1 \%$, which is probably overestimated as the survey was based on a single question (Have you ever experienced symptoms of RLS?). ${ }^{9} 10$ A survey in Japan estimated the incidence of RLS to be $1.5 \%$ in that population. ${ }^{11}$ In India, a survey conducted by Rangarajan et al in Bangalore reported prevalence of $2.1 \%$ with $1.2 \%$ of those surveyed reporting disrupted sleep. ${ }^{12}$ 


\section{CLINICAL FEATURES}

The classical presentation of RLS is the discomfort in the legs that occurs at rest and is relieved by movements. The unpleasant sensations are often described by patients as creeping, crawling, pulling, stretching type of pain, often deep-seated and localised below the knee. These symptoms peak at night and may be severe enough to force the patient to get out of bed for relief of symptoms. The sensory and periodic limb movements of sleep (PLMS) are stereotyped flexion movements of the lower, and sometimes, upper extremities, which last for $0.5-5 \mathrm{~s}$ and occur every 20-40 s throughout sleep and are invariably diagnostic of RLS. The PLMS symptoms are more predominant in the falling phase of core temperature between midnight and $1 \mathrm{am}$ and less between $9 \mathrm{am}$ and $2 \mathrm{pm}$. PLMS symptoms are observed in a majority of patients, and hence, RLS can be viewed as a sensory disease with non-specific motor response, as in PLMS that shares the same underlying mechanism. ${ }^{13}$

The important clinical criterion is the presence of circadian pattern of symptoms occurring in the evening or night and absent in the daytime. ${ }^{3}$ The circadian pattern of symptoms are related to dopaminergic activity that shows natural circadian fluctuations. This association is confirmed by a study by Poceta et al, in which a circadian rhythm for cerebrospinal fluid (CSF) dopamine and homovanillic acid concentrations in humans was demonstrated with higher levels in the daytime than at nighttime, establishing the role of dopamine in the pathophysiology of the disease. ${ }^{14}$

RLS has significant impact on the quality of life of patients. Severe symptoms are associated with psychological impairments related to sleep disruption; poor concentration; and difficulties with work, travel and social events. ${ }^{8}$ As RLS is primarily a subjective disorder, various subjective scales are validated to assess the severity of the disease and its impact on the quality of life. International RLS Rating Scale (IRLS), John Hopkins RLS Severity Scale (JHRLSSS), the RLS-6 scales, and the investigator-based Clinical Global Impressions (CGI) are methods to assess the severity of the disease. The quality of life assessment scales include RLS Quality of Life Instrument (RLS-QLI), Hopkins RLS Quality of Life Questionnaire (RLSQoL), and the RLS Quality of Life Questionnaire (QoL-RLS). In a critical overview on these validated methods by Kohnen et $a l,{ }^{15}$ it was concluded that besides the availability of these methods to investigate the main effects of RLS, there is still a need for the development of methods to address the consequences of RLS on life functioning areas such as ability to work, poor work performance or days missed at work. The objective measurements include polysomnography, periodic limb movement sleep index (PLMI), Periodic limb movement sleep arousal index (PLMS-AI) and efficiency of sleep. ${ }^{16}$

\section{DIAGNOSIS}

The diagnosis of RLS is based on history of the following four diagnostic criteria and physical examination that is invariably normal (unless RLS is associated with secondary conditions). The supportive diagnostic criteria include the presence of positive family history, Periodic leg movements (PLM) and symptomatic relief with dopaminergic therapy. ${ }^{17}$

\section{Diagnostic criteria}

The International RLS Study Group identified four essential diagnostic criteria that constitute the gold standard in the diagnosis of RLS as listed in boxes 1 and 2 .

\section{Box 1 Essential clinical diagnostic criteria ${ }^{18}$}

1. The urge to move the legs that is usually accompanied by unpleasant sensations of leg paraesthesia and dysesthesias

2. The urge to move or unpleasant sensation totally relieved by movements

3. The symptoms that appear are worse at rest and are partially relieved by activity.

4. The symptoms are worse in the evening and during the night.

Supportive diagnostic criteria are helpful when there is uncertainty in the diagnosis of RLS. The presence of these supportive criteria may reinforce the diagnosis as listed in box 2 .

The circadian pattern of symptoms, as discussed, characteristically differentiates RLS from conditions like arthritis, vascular pain syndrome and lumboradicular pain. Recently, a test called the 'Suggested Immobilization Test' (SIT) that supports the clinical diagnosis of RLS was developed. This test has also been used to investigate the circadian pattern of occurrence of RLS symptoms. Martin Michaud et al, in a recent study, investigated the circadian variation of the effects of immobility on symptoms by a $40 \mathrm{~min}$ SIT that was repeated every $2 \mathrm{~h}$ during the $28 \mathrm{~h}$ protocol. The results concluded that worsening of RLS symptoms by immobility is closely linked to their intrinsic circadian variation. $^{19}$

The box 3 lists the common conditions that mimic the symptoms of RLS and their associated clinical features that distinguish them from RLS.

Adapted from Bayard et al. ${ }^{20}$

\section{PATHOPHYSIOLOGY}

Even though the majority of cases of primary RLS appear to be idiopathic in origin, there has been considerable advancement in understanding the pathophysiology of RLS. Secondary RLS is encountered in various underlying medical conditions, namely, iron deficiency anaemia, renal failure, and pregnancy. ${ }^{21}$

\section{Primary RLS}

Genetics

Forty percent of cases of RLS have a positive family history of dominant inheritance, suggesting a genetic basis for this condition. $^{22}$ This syndrome is noted among monozygotic twin pairs with difference in the age and onset of symptoms. ${ }^{23}$ In the molecular genetics level, RLS was demonstrated in eight loci. The five chromosomal positions (12q13-23, 14q13-21, $9 \mathrm{p} 24-22,2 \mathrm{q} 33$, and 20p13) have been determined by modelbased linkage analysis and the recent genome-wide association studies have revealed three genetic loci associated with RLS

\section{Box 2 Supportive diagnostic criteria ${ }^{18}$}

1. Presence of positive family history in primary restless legs syndrome (seen in more than $50 \%$ of patients)

2. Positive response to dopaminergic therapy

3. Sleep disturbances with periodic limb movements in sleep (seen in $85 \%$ of patients) 
Box 3 Differential diagnosis ${ }^{20}$

1. Nocturnal leg cramps: Sudden involuntary muscle contractions associated with palpable tightening of the leg muscles

2. Akathisia: An internal desire to move, most commonly related to the use of neuroleptic medications; desire to move not necessarily associated with discomfort in the legs; and the symptoms are not worse at night

3. Polyneuropathy: Aetiologies include trauma, nerve compression, diabetes, nutritional disorders, infections, others; mostly results in sensory disturbance; may or may not be more noticeable at night; not typically relieved by activity

4. Vascular insufficiency: Primarily a consequence of atherosclerosis; cramping-type pains that are exacerbated by activity and improve with rest; symptoms not worse at night

(6p21.2, 2p 14, and 15q23). ${ }^{24} 25$ The genome-wide association studies also identified variants within the intronic or intergenetic regions of MEIS1 (2p), LBXCOR1/MAP2K5 (15q), BTBD9 (6p), neuronal nitric oxide synthase (NOS1) (12q) and protein tyrosine phosphatase receptor type delta $(9 p)$ genes that suggest a pathological genetic hypothesis for RLS. ${ }^{26}$ The association of these variants in RLS is confirmed by Yang et $a l,{ }^{27}$ in population-based and family-based case control studies in the USA. It is interesting to note that apart from idiopathic RLS, variants in the gene MEIS1 and BTBD9 are demonstrated to be associated with RLS in patients with end-stage renal disease (ESRD). This is the first study that correlated the genetic basis in secondary RLS by Schormair et al..$^{28}$

\section{Dopaminergic system dysfunction}

The symptomatic response with dopamine agonists and levodopa paves the path to prove that dysfunction of the dopaminergic system may play a role in the pathophysiology of the disease. ${ }^{29}$ The role of central dopaminergic system rather than peripheral is corroborated by the fact that administration of peripheral dopamine antagonists does not decrease the efficacy of a dopamine agonist. ${ }^{30}$ The neuroimaging studies with single photon emission computer tomography and positron emission tomography demonstrated a postsynaptic and presynaptic dopamine receptor binding dysfunction at the level of the basal ganglia. Some studies have shown reduced, increased and unchanged dopamine receptor binding, thereby suggesting conflicting evidence of dysfunction of dopamine system. ${ }^{31-34}$ However, the diencephalospinal dopaminergic tract that originates from A11-A14 nuclei is considered as the potential anatomical site of dopaminergic dysfunction. The proximity of this system to the circadian control centres of the hypothalamus may be the cause for the symptomatic changes of circadian pattern diagnostic of RLS. ${ }^{35-37}$

\section{Brain iron metabolism}

There is evidence to suggest that defect in the brain iron metabolism may contribute to the pathogenesis of the disease. This is supported by the fact that iron is a cofactor for tyrosine hydroxylase, which is a rate-limiting step in the conversion of levodopa to dopamine. Therefore decrease in iron may affect the availability of dopamine. It has been identified that there is a circadian variation in the activity of tyrosine hydroxylase that may account for the worsening of symptoms in the night. ${ }^{38}$

Studies by MRI, cerebrospinal fluid and postmortem tissues have shown the role of iron deficiency in the pathogenesis of RLS. MRI studies have demonstrated low iron concentration in the substansia nigra and decrease in ferritin concentrations in the cerebrospinal fluid of patients of RLS. ${ }^{39} 40$ Another study revealed a decrease in transferrin receptor expression in the microvasculature of blood brain interface. ${ }^{41}$ These findings along with the significant response observed after initiation of iron therapy-intravenous or oral-and erythropoietin, further supports the iron-RLS relation. ${ }^{42}{ }^{43}$ An inverse relationship has been suggested between symptom severity and serum ferritin levels finding, which is observed in children as well. ${ }^{44}$

\section{Role of neurotransmitters}

Hypocretin-1 (orexin A) are neurotransmitters produced by the hypothalamus that are essential for normal control of the sleep cycle. They increase arousal and are found to interact with the dopamine system. A study by Allen $e t$ al has identified that there is an increase in the hypocretin levels in the cerebrospinal fluid in the early onset RLS. This was the first study that demonstrated the true difference between patients with early-onset and late-onset RLS. The authors also conclude that elevated levels of hypocretin are responsible for the rare manifestations of sleep deprivation symptoms often observed in these patients. ${ }^{45} 46$

However, a study by Stiasny-Kolster et $a l^{47}$ subsequently failed to observe any difference in hypocretin levels between early-onset and late-onset RLS and the levels at 6 PM were not different in patients with RLS and controls.

\section{Inflammation and immune mechanisms}

The role of inflammation and immune dysfunction was suggested by studies reporting increased prevalence of small intestinal bacterial overgrowth and the case reports of RLS in the presence of infection such as HIV, Streptococcus, mycoplasma, Borrelia and hepatitis $\mathrm{C}$ infection and connective tissue diseases such as systemic lupus erythematosus and Sjogren's syndrome. The three gastrointestinal disorders, namely, Crohn's disease, irritable bowel disease and celiac disease are also associated with RLS. It has been postulated that increase in hepcidin levels may trigger central nervous system (CNS) iron deficiency. Hepcidin is a hormone produced by the liver involved in iron regulation. Hepcidin binds with ferroportin (that export the iron from cells into circulation) and results in its degradation. This creates a state of systemic iron deficiency explaining the decreased iron levels in CSF in these patients. ${ }^{48}$

\section{Secondary RLS}

Secondary RLS appears in later life and encumbers with greater diagnostic challenges, especially in the presence of neurological conditions that might mimic symptoms of RLS. A detailed neurological examination and neurophysiological studies assist in the diagnosis. ${ }^{21}$ Box 4 lists the common causes of secondary RLS.

\section{CLINICAL SIGNIFICANCE OF RLS}

Patients with RLS might have a risk of developing diabetes, hypertension, cardiovascular disease and stroke in the long term. The possible explanation stated is sympathetic hyperactivity due to inadequate diencephalospinal dopaminergic neuron inhibition of sympathetic preganglionic neurons. ${ }^{49}$ However, the Women's Health Study (WHS) and the Physicians' Health 


\section{Box 4 Causes of secondary restless legs syndrome}

\section{- Pregnancy}

- Iron deficiency anaemia

- Venous insufficiency

- Multiple sclerosis

- Parkinsonism

- Rheumatoid arthritis

- Diabetes

- Polyneuropathies

- Alcohol abuse

- Amyotrophic lateral sclerosis

- Vitamin deficiencies

- Spinal stenosis

- Excess caffeine, chocolate intake

- Lumbosacral radiculopathy

- Acute spinal cord lesions-stroke, myelitis

- Hypoglycemia

- Hypothyroidism

- Drugs-tricyclic antidepressants, SSRI antidepressants, antiepileptic drugs, $\beta$ blockers and lithium

- Obesity

Study (PHS), two large prospective cohort studies, do not support these long-term sequelae. ${ }^{50}$

\section{INVESTIGATIONS}

There are no standardised tests that are diagnostic of RLS. Measurement of iron stores with serum ferritin, total iron binding capacity, percentage of transferrin saturation, serum cobalamine (Vit B12), urea, creatinine, and glucose (haemoglobin A1C in established diabetes) can be helpful. Ferreri and Rossini, suggested that electromyography (EMG) and nerve conduction studies should be considered on the clinical grounds even in the presence of normal neurological examination. ${ }^{51}$ Polysomnography is required if there is suspicion of sleep disorder that helps to differentiate RLS from other sleep disorders. $^{13}$

\section{MANAGEMENT}

The following are the goals in the management of RLS:

1. To reduce or cure the troublesome symptoms during rest or sleep

2. Reduce the associated sleep disturbance

3. Reduce daytime fatigue or somnolence

4. Improving the quality of life

\section{Non-pharmacological option}

The non-pharmacological approach includes lifestyle modifications such as avoidance of aggravating factors like alcohol, caffeine and nicotine; mental alertness exercises (video games and reading books); stretching exercises for the posterior leg muscles; and application of heat with warm baths or heating pad. Iron replacement therapy (Ferrous sulfate $325 \mathrm{mg}$ three times daily) can be suggested for patients with low serum ferritin levels (less than $50 \mathrm{ng} / \mathrm{ml}$ ). Treatment of comorbid conditions is advocated in secondary RLS. ${ }^{52}$

\section{Pharmacological therapy}

\section{Dopaminergic agents}

These agents are used in patients who have failed to respond to the non-pharmacological approach. The dopamine agonists available for the treatment of RLS include pramipexole, ropinirole, rotigotine, cabergoline and pergolide. They directly stimulate the dopamine receptors and are generally the drug of choice in view of their lower side effects compared to the dopamine precursors. $^{52}$

The benefits of pramipexole and ropinirole are established in the randomised controlled trials in patients with moderateto-severe RLS and are found to be superior to placebo as assessed by the International Restless Legs Syndrome Study (IRLS) Group rating scale. In a double-blind study by Winkelman et al, pramipexole was found to be superior to placebo in all the three doses of $0.25 \mathrm{mg}, 0.50 \mathrm{mg}$ and $0.75 \mathrm{mg} /$ day. The side effects were low with the dose of $0.25 \mathrm{mg}$ daily. In a long-term follow-up study by Montplaisir et al, 78\% of patients on pramipexole for more than a year, showed improvement in the RLS symptoms, which was sustained in $96 \%$ of them. ${ }^{53} 54$

Ropinirole also demonstrated efficacy in improving the IRLS scores and increasing treatment effect with increasing severity of RLS. The other benefit of ropinirole was improvement in the global symptoms, sleep and quality of life of the patients. The common side effects encountered with these drugs included nausea, vomiting, sedation and orthostatic hypotension. The side effects were mild and transient and usually resolved in 10-14 days. ${ }^{55-58}$

However it needs to be emphasised that augmentation is a known complication of dopaminergic medications, which translates as symptoms that occur at least $2 \mathrm{~h}$ prior to the usual onset of symptoms. During augmentation, the symptoms tend to intensify and spread to previously uninvolved parts of the body such as arms. ${ }^{18}$ Augmentation is also diagnosed in the presence of any of the two following symptoms: increase in symptoms with increase in dosage, decrease in symptoms with decrease in dosage, shortening of therapeutic benefit compared to initiation of therapy and periodic legs movements while awake either presenting or worsening. This phenomenon is probably attributed to excessive dopamine concentration in the CNS resulting in the overstimulation of D1 receptors over the D2 receptors in the spinal cord resulting in D1-related pain and periodic limb movements. Low serum ferritin levels may affect the dopamine transporter and may predispose to augmentation. ${ }^{59}$ The management of augmentation if patient is on levodopa includes gradually tapering the dose and substituting with medications such as dopamine agonists such as ropinirole, pramipexole that may cause augmentation but with less frequency and low intensity. The other options include dividing the drug and providing the lower dose at the earlier time of the day or increasing the total dose and providing the standard dose $1-2 \mathrm{~h}$ prior to the symptom onset, or if unsuccessful with the above strategies, to change to long-acting, high-potency drugs such as methadone or levorphanol. ${ }^{60}$

Although Trenkwalder et al, ${ }^{61}$ reported no augmentation with pramipexole at the end of 9 months of use in 150 patients, there is a risk of augmentation in one-third of patients with long-term use of pramipexole. ${ }^{54}$ Garcia-Borreguero et al reported a $2.3 \%$ incidence of augmentation with ropinirole in their study over a 52 -week period. ${ }^{62}$ This complication is more frequently seen with dopamine precursors such as levodopa, and hence, the dopamine agonists are the preferred drug of choice for RLS. 
Rotigotine is a short-acting dopamine agonist available as transdermal patch. After a prospective open-label, double-blind randomised trial on rotigotine transdermal patch, done over a period of 5 years by Oertel et al, they concluded that rotigotine is safe and well tolerated, and the efficacy is sustained at a stable dose up to 5 years and can be recommended as a treatment option in moderate-to-severe RLS patients who require longterm therapy and especially with daytime symptoms. The common side effects encountered were application site reactions $(58 \%)$ and clinically significant augmentation $(13.2 \%)$ by the end of the 5 -year study. ${ }^{63}$

The dopamine agonist pergolide is currently withdrawn from the USA due to its reported toxicity of restrictive valvular heart disease and retroperitoneal fibrosis. Hence usage of pergolide in the treatment of RLS is contraindicated. ${ }^{64}$ Cabergoline showed high efficacy but its risk of valvular heart disease precludes its recommendation in the routine clinical practice. ${ }^{16}$

Evidence of efficacy of levodopa in the treatment of RLS was proven in small randomised trials and meta-analysis. ${ }^{65}$ Dopamine precursors act by increasing the dopamine concentrations in the brain. Carbidopa is a decarboxylase inhibitor that inhibits the peripheral breakdown of levodopa and does not cross the blood-brain barrier. Although proven effective in RLS, the problems encountered with these agents include augmentation and rebound recurrence of symptoms. ${ }^{66} 67 \mathrm{~A}$ study by Hogl et al showed the risk of augmentation to be $60 \%$ within 6 months of treatment with levodopa with a dose of $300 \mathrm{mg}$. Therefore it is recommended that the maximum dose of levodopa should not be exceeded beyond $300 \mathrm{mg} .{ }^{68}$ The possibility of augmentation should be discussed with all patients and they should be advised against altering the dosage of the medication without their physician's advice. Therefore according to the expert advisory panel on RLS, levodopa is recommended only in patients with intermittent RLS, which is defined as symptoms that are troublesome enough to require intermittent therapy but not frequent enough for daily therapy. ${ }^{16}$

\section{Benzodiazepines}

Clonazepam is one of the first few earlier drugs that were considered in the treatment of RLS. ${ }^{1}$ As was demonstrated by Saletu et al, it tends to improve sleep and reduce arousals due to PLMS but it was not effective in reducing the motor and sensory abnormalities associated with RLS. ${ }^{69}$ Hence it was concluded that clonazepam had therapeutic effect on insomnia but not on the limb movements. ${ }^{16}$ Short-acting agents like triazolam, zalepon and temazepam, zolpidem were effective for sleep onset insomnia but the intermediate acting agent like temazepan was recommended if the patient awakens later at night. ${ }^{52}$ Overall, evidence recommends clonazepam as an option as an adjunctive medication.

\section{Opioids}

Opioids decrease the release of neurotransmitters resulting in analgesic effect. There is low level of evidence reporting the benefit of opioids as first-line treatment for RLS. In a doubleblind study by Walters et al, opioids were found to be superior to placebo in reducing the frequency of PLMS, sleep arousals and sleep disturbances associated with RLS. However, they require monitoring for sleep apnoea either clinically or by polysomnography. ${ }^{70}$ One should be aware that the risk of abuse with opioids is of undefined potential in predisposed patients. ${ }^{16}$ The major side effects are nausea and constipation. In a study by Silver et al, a 10-year retrospective review of 76 patients on methadone, no augmentation was reported at the end of the study. In general, these drugs are well tolerated and the indication of opioids is as second-line agents in patients with augmentation, non-responders to dopamine therapy and in refractory RLS. ${ }^{71}$

\section{Anticonvulsants}

Gabapentin was effective in the treatment of mild-to-moderate RLS as shown by Happe et al and Garcia-Borreguero et al. There was significant improvement in the PLMS and sleep architecture. It was identified that patients with pain, experienced relief with gabapentin. However, there were concerns regarding the side effects such as sedation, dizziness and suicidal tendencies. $^{72} 73$

Studies demonstrated improvement in the symptoms of RLS including sleep architecture and mood with gabapentin enacarbil. The common adverse effects observed were mild somnolence and dizziness. ${ }^{74-76}$ In a retrospective analysis by Ellenbogen et al there were no reports on augmentation or rebound symptoms or impulsive behaviour disorders. ${ }^{77}$ With the present encouraging evidence of good tolerability and low side-effect profile, gabapentin enacarbil is recommended for therapy for RLS. ${ }^{16}$

Two studies on the use of pregabalin ( $\alpha 2$ delta ligand) demonstrated its effectiveness in the treatment of moderate-to-severe RLS. Allen et al reported a 90\% reduction in RLS symptoms and Garcia-Borreguero et al reported that patients on pregabalin $(300 \mathrm{mg} /$ day) had a $2.1 \%$ incidence of augmentation. The latter study revealed that a non-dopaminergic drug had a low incidence of augmentation. ${ }^{78} 79$

\section{Other drugs}

Although carbamazepine was effective, side effects like sedation and liver function abnormalities prevented it from being used for therapy of RLS. ${ }^{16}$ Iron supplementation was shown to be effective in the treatment of RLS patients who had low serum ferritin. Wang et al reported statistically significant improvement in patients with low serum ferritin levels with two doses of $325 \mathrm{mg} /$ day of oral ferrous sulfate. ${ }^{80}$ However, Earley et al and Grote et al reported no effect with iron sucrose, and inconsistent results with the use of iron dextran. ${ }^{81-83} 42$

RLS symptoms were relieved with iron supplementation when ferritin levels were less than $50 \mathrm{ng} / \mathrm{ml}$ but there was no evidence of improvement of symptoms in patients with normal ferritin levels. ${ }^{83}$ It can be concluded that oral iron is poorly absorbed in the presence of normal body iron stores. In another study by the same author, Earley et al, in 2005, the efficacy and safety of repeated iron infusions was evaluated in RLS patients who initially demonstrated response to a single dose of iron dextran. The study revealed that supplemental iron treatments can sustain initial improvement, but not the high ferritin levels achieved after a single dose IV iron treatment. The authors highlight that rapid iron loss is probably related to pathophysiology of RLS. The data from the study therefore suggest that repeated IV dosing of iron is required to maintain treatment effectiveness. ${ }^{84}$

\section{RLS IN PREGNANCY}

The prevalence of RLS in pregnancy, as is evident from the epidemiological data, is around $11-27 \%$ with the risk of it being two to three times higher in pregnancy than the given general population. The aetiology is most likely due to altered iron metabolism during pregnancy, thereby supporting the concept of iron deficiency as the cause of RLS. The peak incidence is seen in the third trimester, which resolves after delivery. However, the risk of recurrence is $30 \%$ in subsequent pregnancies. $^{85} 86$ 
The aim of treatment in pregnancy is to provide symptomatic relief and improve the quality of life. Non-pharmacological measures are usually advocated in mild cases and drugs are initiated only in moderate-to-severe cases. Non-pharmacological therapy like leg stretching before sleep and use of elastic stockings when associated with varicose veins have proven to be beneficial. $^{52}$

\section{PHARMACOLOGICAL THERAPY IN PREGNANCY Dopaminergic agents}

There is paucity of data on the use of dopamine agonists in pregnancy. However, there is a case report by Mucchiut et al on the use of pramipexole in pregnancy with parkinsonism, which did not show any adverse outcome. Levodopa appears to be safe in pregnancy according to the scanty evidences available. ${ }^{87} 88$

\section{Opioids}

There is evidence supporting the use and safety of opioids in pregnancy. The concerns are neonatal respiratory depression, especially if used in late pregnancy. Walters et $a l^{89}$ had considered these agents to be effective for long-term treatment with no evidence of tolerance or addiction.

\section{Benzodiazepines}

Benzodiazepines are considered to be effective in treating RLS in pregnancy. The concerns of risk of cleft palate, especially if used after first trimester, were shown to be negligible in a meta-analysis by Dolovich et al. Although of rare occurrence, neonatal withdrawal syndrome has been reported. The benzodiazepine of choice is clonazepam. Others such as temazepam and triazolam were also found to be effective. ${ }^{9091}$

\section{Anticonvulsants}

Carbamazepine is the first anticonvulsant evaluated in the treatment of RLS. With the safety data and proven efficacy in RLS, it is a reasonable option to be considered in pregnancy. The data on gabapentin were also reassuring in terms of safety to the mother and foetus. One should also note that all anticonvulsants are associated with risk of neural tube defects in pregnancy, and hence, there is a need for continuing folic acid (5 mg) supplementation from the first trimester onwards. ${ }^{92} 93$

\section{Maternal and foetal problems}

Miri $S$ et al concluded that secondary RLS was associated low haemoglobin levels than idiopathic RLS $(p=0.004)$. However, duration of sleep and birth weight of neonates were significantly reduced in idiopathic RLS ( $p=0.006$ and $p=0.039$ respectively). There was no difference in both the groups in terms of positive family history, parity, duration of pregnancy and rate of Caesarean section. The authors surmised that idiopathic RLS can have a negative effect during pregnancy, and hence, require screening for the condition to optimise outcome. ${ }^{94}$

\section{Management of other secondary RLS}

The prevalence of RLS in haemodialysis patient is 6-60\%. It appears to be more severe than primary RLS and is a predictor of mortality in these patients. ${ }^{95} 96$ RLS in ESRD may be related to anaemia as benefit was observed with low-dose erythropoietin and renal transplantation. ${ }^{16}$ In a randomised control study by Thorpe $e t$ al, considerable benefits were observed with gabapentin. ${ }^{97}$ The prevalence of RLS is highest among patients with parkinsonism. The long-term anti-parkinsonism treatment results in development of RLS. This may be attributed to the wearing-off episodes of levodopa that mimics symptoms of RLS or the augmentation effects of levodopa. ${ }^{98} 99$ Varicose veins are associated with RLS and sclerotherapy in these patients was associated with relief in $98 \%$ of these patients. Treatment with hydroxyethylrutoside in these patients demonstrated relief although not as dramatic as with sclerotherapy. ${ }^{100}{ }^{101}$ Assessing serum ferritin levels and treatment of iron deficiency is of value. ${ }^{102}$ It is prudent to also incorporate lifestyle changes such as exercise, avoidance of caffeine and appropriate sleep habits along with the drugs.

\section{CONCLUSIONS}

There is considerable amount of advancement made in the understanding of the pathophysiological aspects and therapeutic management of this syndrome. The pathophysiology is centred on dopaminergic system dysfunction, altered iron hemostasis with decreased CNS iron, associated genetic linkages, or alteration in the neurotransmitters levels, like in hypocretins and endorphins. A new insight into this multifactorial pathophysiology is the proposed role of immune dysfunction and inflammation in the evolution of this disease process, which is richly supported by evidence. With the wide variety of pharmacological and non-pharmacological options available, it is recommended that the clinician be updated in standardising the prescription practices for symptomatic relief and to improve the quality of life in these patients. There are strong recommendations for dopamine agonists such as rotigotine transdermal patch for which long-term studies up to 5 years are available. A randomised control study has showed that pregabalin had significantly low level of augmentation. Levodopa, given the risk of augmentation, which is higher compared to dopamine agonists, has a low level of recommendation. Based on expert consensus, dopamine agonists or gabapentin or low-potency opioids are the initial choices. For intermittent RLS, the expert panel recommends levodopa or dopamine agonists or lowpotency opioids or benzodiazepines. In refractory RLS, the choice is to change to gabapentin or a different dopamine agonist, addition of a second agent like gabapentin or benzodiazepine to the existing drug or changing to high-potency opioid or tramadol. There are concerns that patients with RLS are at risk of metabolic deregulation, autonomic dysfunction and cardiovascular morbidity. A recent study concluded that men or women with RLS are not at increased risk of cardiovascular complications though we need more evidence to affirm it.

\section{Main messages}

- Restless legs syndrome (RLS) defined by the essential criteria: (a)an urge to move the legs usually but not always associated with leg discomfort; (b) worsening of symptoms at rest, either lying or sitting; (c) partial and temporary relief of symptoms by activity; (d) worsening of symptoms later in the day or at night.

- The understanding in the pathophysiology of RLS is dopaminergic dysfunction, systemic iron deficiency, genetic linkage and more recent postulation related to inflammation and immune dysfunction.

- Dopamine agonists are currently the first line of agents in the treatment of idiopathic RLS; however, if supported with more long-term trials with other non-dopaminergic agents, the recommendation practices may change in the future. 
Current research questions

- There is a need to perform prospective studies of iron deficiency, small intestinal bacterial overgrowth and immune and inflammatory mechanisms contributing to primary and secondary RLS.

- More comparative long-term trials are required between the different classes of drugs that would help to determine the efficacy and augmentation rates more effectively.

- The treatment options available for idiopathic RLS do not have sufficient evidence in the management of secondary RLS. This highlights the need for randomised controlled trials to evaluate the treatment options for secondary RLS.

\section{Key references}

- Aurora RN; Kristo DA; Bista SR, et al. The treatment of restless legs syndrome and periodic limb movement disorder in adults - an update for 2012: practice parameters with an evidence-based systematic review and meta-analyses. Sleep 2012;35:1039-1062

- Garcia-Borreguero D, Ferini-Strambi L, Kohnen R, et al. European guidelines on management of restless legs syndrome: report of a joint task force by the European Federation of Neurological Societies, the European Neurological Society and the European Sleep Research Society. Eur J Neurol 2012;11:1385-96.

- Silber MH, Ehrenberg BL, Allen RP, et al. An algorithm for the management of restless legs syndrome. Mayo Clin Proc 2004;79:916.

- Sforza E, Mathis J, Bassetti CL. Restless legs syndrome: pathophysiology and clinical aspects. Schweiz Arch Neurol Psychiatr 2003;154:349-57.

- Allen RP, Picchietti D, Hening WA, et al. Restless legs syndrome: diagnostic criteria, special considerations, and epidemiology. A report from the restless legs syndrome diagnosis and epidemiology workshop at the National Institutes of Health. Sleep Med 2003;4:101-19.

\section{Self assesment questions}

1. In the essential criteria for RLS-The urge to move the legs that is always accompanied by unpleasant sensations of leg paraesthesia and dyaesthesias

2. The dopaminergic agents levodopa/carbidopa are the first line of agents in the treatment of RLS

3. RLS symptoms are relieved with iron supplementation with ferritin levels less than $50 \mathrm{ng}$ per $\mathrm{ml}$

4. In patients with ESRD, RLS appears to be more severe and is a predictor of mortality in these patients

5. Recent evidence reports low augmentation risk with pregablin over other dopaminergic agents.
Collaborators Dr Sachchithanantham Kanagasabai.

Competing interests None.

Provenance and peer review Not commissioned; externally peer reviewed.

\section{REFERENCES}

1 Garcia-Borreguero D, Ferini-Strambi L, Kohnen R, et al. European guidelines on management of restless legs syndrome: report of a joint task force by the European Federation of Neurological Societies, the European Neurological Society and the European Sleep Research Society. Eur J Neurol 2012;11:1385-96.

2 Benes $\mathrm{H}$, Walters AS, Allen RP, et al. Definition of restless legs syndrome, how to diagnose it, and how to differentiate it from RLS mimics. Mov Disord 2007;22 (Suppl 18):S401-8.

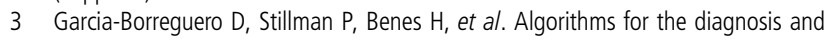
treatment of restless legs syndrome in primary care. BMC Neurology 2011;11:28.

4 Högl B, Kiechl S, Willeit J, et al. Restless legs syndrome: a community-based study of prevalence, severity, and risk factors. Neurology 2005;64:1920.

5 Allen RP, Bharmal M, Calloway M. Prevalence and disease burden of primary restless legs syndrome: results of a general population survey in the United States. Mov Disord 2011;26:114

6 Hening W, Walters AS, Allen RP, et al. Impact, diagnosis and treatment of restless legs syndrome (RLS) in a primary care population: the REST (RLS epidemiology, symptoms, and treatment) primary care study. Sleep Med 2004;5:237-46.

7 Hanson $\mathrm{M}$, Honour $\mathrm{M}$, Singleton $\mathrm{A}$, et al. Analysis of familial and sporadic restless legs syndrome in age of onset, gender, and severity features. J Neurol 2004;251:1398-401.

8 Allen RP, Walters AS, Montplaisir J, et al. Restless legs syndrome prevalence and impact: REST general population study. Arch Intern Med 2005;165:1286.

9 Tan EK, Seah A, See SJ, et al. Restless legs syndrome in an Asian population: a study in Singapore. Mov Disord 2001;16:577-9.

10 Kim J, Choi C, Shin K, et al. Prevalence of restless legs syndrome and associated factors in the Korean adult population: the Korean Health and Genome Study. Psychiatry Clin Neurosci 2005;59:350-53.

11 Kageyama T, Kabuto M, Nitta $H$, et al. Prevalence of periodic limb movement-like and restless legs-like symptoms among Japanese adults. Psychiatry Clin Neurosci 2000;54:296-8

12 Rangarajan S, Rangarajan S, D'Souza GA. Restless legs syndrome in an Indian urban population. Sleep Med 2007;9:88-93.

13 Sforza E, Mathis J, Bassetti CL. Restless legs syndrome: pathophysiology and clinical aspects. Schweiz Arch Neurol Psychiatr 2003;154:349-57.

14 Poceta JS, Parsons L, Engelland S, et al. Circadian rhythm of CSF monoamines and hypocretin-1 in restless legs syndrome and Parkinson's disease. Sleep Med 2009;10:129-33.

15 Kohnen $\mathrm{R}$, Allen RP, Benes $\mathrm{H}$, et al. Assessment of restless legs syndromemethodological approaches for use in practice and clinical trials. Mov Disord 2008:8:1200-2.

16 Aurora RN, Kristo DA, Bista SR, et al. The treatment of restless legs syndrome and periodic limb movement disorder in adults - an update for 2012: practice parameters with an evidence-based systematic review and meta-analyses. SLEEP 2012;35:1039-62.

17 Inoue $Y$. Diagnosis and symptom rating scale of restless legs syndrome. Brain Nerve 2009:5:533-8.

18 Allen RP, Picchietti D, Hening WA, et al. Restless legs syndrome: diagnostic criteria, special considerations, and epidemiology. A report from the restless legs syndrome diagnosis and epidemiology workshop at the National Institutes of Health. Sleep Med 2003;4:101-19.

19 Michaud M, Dumont M, Paquet J, et al. Circadian variation of the effects of immobility on symptoms of restless legs syndrome. Sleep 2005;7:843-6.

20 Bayard M, Avonda T, Wadzinski J. Restless legs syndrome. Am Fam Physician 2008;78:235-40.

21 Oka Y, loue Y. Secondary restless legs syndrome. Brain Nerve 2009;61:539-47.

22 Montplaisir J, Boucher S, Poirier G, et al. Clinical, polysomnographic, and genetic characteristics of restless legs syndrome: a study of 133 patients diagnosed with new standard criteria. Mov Disord 1997;12:61.

23 Ondo WG, Vuong KD, Wang Q. Restless legs syndrome in monozygotic twins: clinical correlates. Neurology 2000;55:1404.

24 Pichler I, Hicks AA, Pramstaller PP. Restless legs syndrome: an update on genetics and future perspectives. Clin Genet 2008;73:297-305.

25 Fan A, Rao SQ. Advances in genetics of restless legs syndrome. Yi Chuan. 2009;31:675-82

26 Trenkwalder C, Högl B, Winkelmann . Recent advances in the diagnosis, genetics and treatment of restless legs syndrome. J Neurol. 2009;256:539-53.

27 Yang Q, Li L, Chen Q, et al Association studies of variants in MEIS1, BTBD9, and MAP2K5/SKOR1 with restless legs syndrome in a US population. Sleep Med 2011:12:800-4.

28 Schormair B, Plag J, Kaffe M, et al. MEIS1 and BTBD9: genetic association with restless leg syndrome in end stage renal disease. J Med Genet 2011:48:462-6. 
29 Rye DB, Adler CH, Allen RP, et al. RLS medical bulletin. Rochester, MN: Restless Legs Syndrome Foundation, 2005.

30 Wetter TC, Stiasny K, Winkelmann J, et al. A randomized controlled study of pergolide in patients with restless legs syndrome. Neurology 1999;52:944-50.

31 Ruottinen HM, Partinen M, Hublin C, et al. An FDOPA PET study in patients with periodic limb movement disorder and restless legs syndrome. Neurology 2000;54:502.

32 Turjanski N, Lees AJ, Brooks DJ. Striatal dopaminergic function in restless legs syndrome: 18F-dopa and 11C-raclopride PET studies. Neurology 1999;52:932.

33 Cervenka S, Pålhagen SE, Comley RA, et al. Support for dopaminergic hypoactivity in restless legs syndrome: a PET study on D2-receptor binding. Brain 2006;129:2017

34 Eisensehr I, Wetter TC, Linke $\mathrm{R}$, et al. Normal IPT and IBZM SPECT in drug-naive and levodopa-treated idiopathic restless legs syndrome. Neurology 2001;57:1307.

35 Ondo WG. Restless Legs Syndrome. In: Jankovic J, Tolosa E. Parkinson's disease and movement disorders. 5th edn. Philidelphia: Lippincott, Williams, and Wilkins, 2007:409-20

36 Trenkwalder C, Paulus W, Walters AS. The restless legs syndrome. Lancet Neurol 2005:4:465-75.

37 Earley CJ, Allen RP, Beard JL, et al. Insight into the pathophysiology of restless legs syndrome. J Neurosci Res 2000;62:623-8.

38 Allen RP, Earley CJ. The role of iron in restless legs syndrome. Mov Disord 2007;22:S440-8.

39 Earley CJ, Connor JR, Beard JL, et al. Abnormalities in CSF concentrations of ferritin and transferrin in restless legs syndrome. Neurology 2000;54:1698-700.

40 Allen RP, Barker PB, Wehrl F, et al. MRI measurement of brain iron in patients with restless legs syndrome. Neurology 2001;56:263-5.

41 Krieger J, Schroeder C. Iron, brain and restless legs syndrome. Sleep Med Rev 2001;5:277-86

42 Earley CJ, Heckler D, Allen RP. The treatment of restless legs syndrome with intravenous iron dextran. Sleep Med 2004;5:231-5.

43 Kemlink D, Sonka K, Pretl M, et al. Suggestive evidence of erythropoietin level abnormality in patients with sporadic and familial cases of the restless legs syndrome. Neuro endocrinology letters 2007;28:643-6.

44 Allen RP. Race, iron status and restless legs syndrome. Sleep Med 2002:3:467-8.

45 Taheri S, Zeitzer JM, Mignot E. The role of hypocretins (orexins) in sleep regulation and narcolepsy. Annu Rev Neurosci 2002;25:283.

46 Allen RP, Mignot E, Ripley B, et al. Increased CSF hypocretin-1 (orexin-A) in restless legs syndrome. Neurology 2002;59:639.

47 Stiasny-Kolster K, Mignot E, Ling L, et al. CSF hypocretin-1 levels in restless legs syndrome. WH Neurology 2003;10:1426-9.

48 Weinstock LB, Walters AS, Paueksakon P. Restless legs syndrome-theoretical roles of inflammatory and immune mechanisms. Sleep Med Rev 2012;6:341-54.

49 Walters AS, Rye DB. Review of the relationship of restless legs syndrome and periodic limb movements in sleep to hypertension, heart disease, and stroke. Sleep 2009:32:589-97.

50 Winter AC, Schürks M, Glynn RJ, et al. Restless legs syndrome and risk of incident cardiovascular disease in women and men: prospective cohort. BMJ Open 2012;2:e000866.

51 Ferreri F, Rossini PM. Neurophysiological investigations in restless legs syndrome and other disorders of movement during sleep. Sleep Med 2004;4:397-9.

52 Silber MH, Ehrenberg BL, Allen RP, et al. An algorithm for the management of restless legs syndrome. Mayo Clin Proc 2004;79:916.

53 Winkelman JW, Sethi KD, Kushida CA, et al. Efficacy and safety of pramipexole in restless legs syndrome. Neurology 2006;67:1034.

54 Montplaisir J, Fantini ML, Desautels A, et al. Long-term treatment with pramipexole in restless legs syndrome. Eur J Neurol 2006;13:1306-11.

55 Bogan R, Fry J, Schmidt M, et al TREAT RLS US (Therapy with Ropinirole Efficacy and Tolerability in RLS US) Study Group. Ropinirole in the treatment of patients with restless legs syndrome: a US-based randomized, double-blind, placebo-controlled clinical trial. Mayo Clin Proc 2006;81:17-27.

56 Trenkwalder C, Garcia-Borreguero D, Montagna P, et al. Ropinirole in the treatment of restless legs syndrome: Results from the TREAT RLS 1 study, a 12-week, randomised, placebo-controlled study in 10 European countries. J Neurol Neurosurg Psychiatry 2004;75:92-7.

57 Walters A, Ondo W, Dreykluft $\mathrm{T}$, et al. Ropinirole is effective in the treatment of restless legs syndrome. TREAT RLS 2: A 12-week, double-blind, randomized, parallel-group, placebo-controlled study. Mov Disord 2004;19:1414-23.

58 Allen R, Becker P, Bogan R, et al. Ropinirole decreases periodic leg movements and improves sleep parameters in patients with restless legs syndrome. Sleep 2004:27:907-14.

59 Paulus W, Trenkwalder C. Less is more: pathophysiology of dopaminergic-therapy-related augmentation in restless legs syndrome. Lancet Neurol. 2006;5:878-86.

60 Teofilo L. Lee-Chiong. Sleep: a comprehensive handbook. USA: John Wiley and Sons, 2006:473-80.

61 Trenkwalder C, Stiasny-Kolster K, Kupsch A, et al. Controlled withdrawal of pramipexole after 6 months of open-label treatment in patients with restless legs syndrome. Mov Disord 2006:21:1404-10.
62 Garcia-Borreguero D, Grunstein R, Sridhar G, et al. A 52-week open label study of the long-term safety of ropinirole in patients with restless legs syndrome. Sleep Med 2007:8:742-52.

63 Oertel W, Trenkwalder C, Beneš H, et al. Long-term safety and efficacy of rotigotine transdermal patch for moderate-to-severe idiopathic restless legs syndrome: a 5-year open-label extension study. Lancet Neurol 2011;10:710-20.

64 Van Camp G, Flamez A, Cosyns B, et al. Treatment of Parkinson's disease with pergolide and relation to restrictive valvular heart disease. Lancet 2004;363:1179.

65 Scholz H, Trenkwalder C, Kohnen R, et al. Levodopa for restless legs syndrome. Cochrane Database Syst Rev 2011, Issue 2. Art:: CD005504.

66 Allen RP, Earley CJ. Augmentation of the restless legs syndrome with carbidopa/ levodopa. Sleep 1996:19:205-13.

67 Earley CJ, Allen RP. Pergolide and carbidopa/levodopa treatment of the restless legs syndrome and periodic leg movements in sleep in a consecutive series of patients. Sleep 1996;19:801.

68 Hogl B, Garcia-Borreguero D, Kohnen R, et al. Progressive development of augmentation during long-term treatment with levodopa in restless legs syndrome: results of a prospective multi-center study. J Neurol 2010;257:230-7.

69 Saletu M, Anderer P, Saletu-Zyhlarz G, et al. Restless legs syndrome (RLS) and periodic limb movement disorder (PLMD) Acute placebo controlled sleep laboratory studies with clonazepam. Eur Neuropsychopharmacol 2001;11:153-61.

70 Walters A, Winkelman J, Trenkwalder C, et al. Long-term follow-up on restless legs syndrome patients treated with opioids. Mov Disord 2001;16:1105-9.

71 Silver N, Allen RP, Senerth J, et al. A 10-year, longitudinal assessment of dopamine agonists and methadone in the treatment of restless legs syndrome. Sleep Med 2011;5:440-4

72 Happe S, Sauter C, Klosch G, et al. The Treatment of restless legs syndrome: gabapentin versus ropinirole in the treatment of idiopathic restless legs syndrome. Neuropsychobiology 2003;48:82-6.

73 Garcia-Borreguero D, Larrosa 0 , de la Llave $Y$, et al. Treatment of restless legs syndrome with gabapentin: a double-blind, cross-over study. Neurology 2002:59:1573-9.

74 Kushida CA, Walters AS, Becker $P$, et al. A randomized, double-blind, placebo-controlled, crossover study of XP13512/GSK1838262 in the treatment of patients with primary restless legs syndrome. Sleep 2009;32:159-68.

75 Walters AS, Ondo WG, Kushida CA, et al. Gabapentin enacarbil in restless legs syndrome: a phase $2 \mathrm{~b}, 2$-week, randomized, double-blind, placebo-controlled trial. Clin Neuropharmacol 2009:32:311-20.

76 Lee DO, Ziman MD, Perkins AT, et al. A randomized, double-blind, placebo-controlled study to assess the efficacy and tolerability of gabapentin enacarbil in subjects with restless legs syndrome. J Clin Sleep Med 2011;7:282-92.

77 Ellenbogen AL, Thein SG, Winslow D, et al. A 52-week study of gabapentin enacarbil in restless legs syndrome. Clin Neuropharmacol 2011;34:8-16.

78 Allen R, Chen C, Soaita A, et al. A randomized, double-blind, 6-week, dose-ranging study of pregabalin in patients with restless legs syndrome. Sleep Med 2010;11:512-19.

79 Garcia-Borreguero D, Crystal chew MD, Allen R, et al. Long-term efficacy and augmentation assessment of a dopamine agonist (pramipexole) compared with an alpha-2-delta ligand (pregabalin) in restless legs syndrome: results of a randomized, double-blinded, placebo-controlled trial. Abstract 0012012 Emerg Sci Abstr Neurol 2012;79:e87-91.

80 Wang J, O'Reilly B, Venkataraman R, et al Efficacy of oral iron in patients with restless legs syndrome and a low-normal ferritin: a randomized, double-blind, placebo-controlled study. Sleep Med 2009;10:973-5.

81 Earley CJ, Horska A, Mohamed MA, et al. A randomized, double-blind, placebo-controlled trial of intravenous iron sucrose in restless legs syndrome. Sleep Med 2009;10:206-11.

82 Grote L, Leissner L, Hedner J, et al. A randomized, double-blind, placebo controlled, multi-center study of intravenous iron sucrose and placebo in the treatment of restless legs syndrome. Mov Disord 2009;24:1445-52.

83 Bayard M, Avonda T, Wadzinski J. Restless leg syndrome. Am Fam Physician 2008;78:235-40.

84 Goodman JD, Brodie C, Ayida GA. Restless leg syndrome in pregnancy. BM 1988;297:1101-2

85 Earley CJ, Heckler D, Allen RP. Repeated IV doses of Iron provides effective supplemental treatment of restless leg syndrome. Sleep Med 2005;4:301-5

86 Manconi M, Govoni V, De Vito A, et al. Pregnancy as a risk factor for restless legs syndrome. Sleep Med 2004;5:305-8.

87 Mucchiut M, Belgrado E, Cutuli D, et al. Pramipexole-treated Parkinson's disease during pregnancy. Mov Disord 2004;19:1114-15.

88 Scott M, Chowdhury M. Pregnancy in Parkinson's disease: unique case report and review of the literature. Mov Disord 2005:20:1078-9.

89 Walters AS, Winkelmann J, Trenkwalder C, et al. Long-term follow-up on restless legs syndrome patients treated with opioids. Mov Disord 2001;16:1105-9.

90 Dolovich LR, Addis A, Vaillancourt JM, et al. Benzodiazepine use in pregnancy and major malformations or oral cleft: meta-analysis of cohort and case-control studies. BMJ 1998:317:839-43. 
91 Saletu M, Anderer P, Saletu-Zyhlarz G, et al. Restless legs syndrome (RLS) and periodic limb movement disorder (PLMD): acute placebo-controlled sleep laboratory studies with clonazepam. Eur Neuropsychopharmacol 2001;11:153-61.

92 Telstad W, Sørensen O, Larsen S, et al. Treatment of the restless legs syndrome with carbamazepine: a double blind study. BMJ 1984;288:444-6.

93 Montouris $G$. Gabapentin exposure in human pregnancy: results from the Gabapentin Pregnancy Registry. Epilepsy Behav 2003:4:310-17.

94 Miri S, Vahdat M, Sariri E, et al. A comparative study of idiopathic and secondary restless legs syndrome in pregnancy [abstract]. Mov Disord 2012;27:1222.

95 Saudi J. Restless legs syndrome in patients on hemodialysis. Kidney Dis Transp/ 2011;22:368-72

96 Kavanagh D, Siddiqui S, Geddes CC. Restless legs syndrome in patients on dialysis. Am J Kidney Dis 2004;43:763.

97 Thorpe ML, Morris CD, Bagby SP. A crossover study of gabapentin in treatment of restless legs syndrome among hemodialysis patients. Am J Kidney Dis 2001;38:104-8.

98 Lee JE, Shin HW, Kim KS, et al. Factors contributing to the development of restless legs syndrome in patients with Parkinson disease. Mov Disord 2009;24:579.

99 Peralta CM, Frauscher B, Seppi K, et al. Restless legs syndrome in Parkinson's disease. Mov Disord 2009;24:2076.
100 Kanter AH. The effect of sclerotherapy on restless legs syndrome. Dermatol Surg 1995;21:328.

101 Poynard T, Valterio C. Meta-analysis of hydroxyethylrutosides in the treatment of chronic venous insufficiency. Vasa 1994;23:244.

102 Konofal $\mathrm{E}$, Cortese $\mathrm{S}$, Marchand $\mathrm{M}$, et al. Impact of restless legs syndrome and iron deficiency on attention-deficit/hyperactivity disorder in children. Sleep Med 2007;8:711-15.

\section{Self assesment answers}
1. $\mathbf{F}$
2. $F$
3. $\mathbf{T}$
4. $\mathrm{T}$
5. $\mathbf{T}$

\section{Warwick University short courses}

\section{7-20 September 2013}

\section{Management of CKD}

A four day course introducing the underlying scientific background, pathophysiology and clinical management of people with chronic kidney disease. Optional accreditation leads to a masters level Postgraduate Award.

For further details please contact Dr Charlotte Moonan via Charlotte.Moonan@warwick.ac.uk or go to the course web site http://www2.warwick.ac.uk/fac/sci/lifesci/study/shortcourses/kidney/ckd/

\section{8-21 March 2014}

\section{The Kidney as an Endocrine Organ}

A four day course providing a comprehensive insight into the role of the kidney as an endocrine organ. The course will introduce the basic physiology of the main endocrine functions of the kidney and clinical conditions associated with abnormal function, including hypertension, diabetes mellitus and chronic kidney disease. These two elements are integrated throughout the course, particularly during the small group work. Optional accreditation leads to a masters level Postgraduate Award.

For further details please contact Dr Charlotte Moonan via Charlotte.Moonan@warwick.ac.uk or go to the course web site http://www2.warwick.ac.uk/fac/sci/lifesci/study/shortcourses/kidney/keo/ 